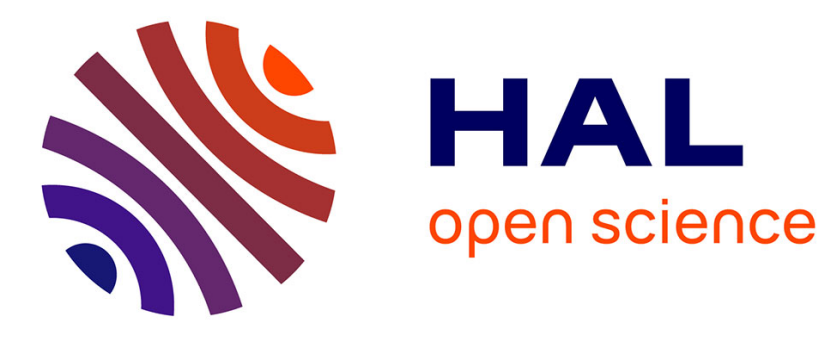

\title{
Sojourn time approximations in a multi-class time-sharing server
}

Ane Izagirre, Urtzi Ayesta, Maaike Verloop

\section{To cite this version:}

Ane Izagirre, Urtzi Ayesta, Maaike Verloop. Sojourn time approximations in a multi-class time-sharing server. IEEE International conference on Comptuer Communications - INFOCOM 2014, Apr 2014, Toronto, Canada. pp. 2786-2794. hal-01147319

\section{HAL Id: hal-01147319 https://hal.science/hal-01147319}

Submitted on 30 Apr 2015

HAL is a multi-disciplinary open access archive for the deposit and dissemination of scientific research documents, whether they are published or not. The documents may come from teaching and research institutions in France or abroad, or from public or private research centers.
L'archive ouverte pluridisciplinaire HAL, est destinée au dépôt et à la diffusion de documents scientifiques de niveau recherche, publiés ou non, émanant des établissements d'enseignement et de recherche français ou étrangers, des laboratoires publics ou privés. 


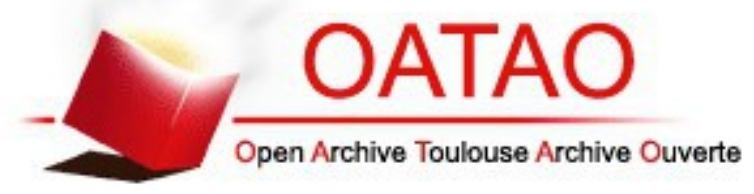

\section{Open Archive TOULOUSE Archive Ouverte (OATAO)}

OATAO is an open access repository that collects the work of Toulouse researchers and makes it freely available over the web where possible.

This is an author-deposited version published in : http://oatao.univ-toulouse.fr/ Eprints ID : 12817

To link to this article : DOI :10.1109/INFOCOM.2014.6848228

URL : http://dx.doi.org/10.1109/INFOCOM.2014.6848228

To cite this version : Izagirre, Ane and Ayesta, Urtzi and Verloop, Maaike Sojourn time approximations in a multi-class time-sharing server. (2014) In: IEEE International conference on Comptuer Communications - INFOCOM 2014, 27 April 2014 - 2 May 2014 (Toronto, Canada).

Any correspondance concerning this service should be sent to the repository administrator: staff-oatao@,listes-diff.inp-toulouse.fr 


\title{
Sojourn time approximations in a multi-class time-sharing server
}

\author{
A. Izagirre ${ }^{b, e}$, U. Ayesta ${ }^{b, c, d, e}$, I.M. Verloop ${ }^{a, e}$ \\ ${ }^{a}$ CNRS, IRIT, 2 rue C. Camichel, 31071 Toulouse, France \\ ${ }^{b}$ CNRS, LAAS, 7 avenue du colonel Roche, 31400 Toulouse, France \\ ${ }^{c}$ IKERBASQUE, Basque Foundation for Science, 48011 Bilbao, Spain \\ ${ }^{d}$ UPV/EHU, Univ. of the Basque Country, 20018 Donostia, Spain \\ ${ }^{e}$ Univ. de Toulouse, INP, INSA, LAAS, 31400 Toulouse, France
}

\begin{abstract}
We study a multi-class time-sharing discipline with relative priorities known as Discriminatory Processor Sharing (DPS), which provides a natural framework to model service differentiation in systems. The analysis of DPS is extremely challenging and analytical results are scarce. We develop closedform approximations for the mean conditional and unconditional sojourn times. The main benefits of the approximations lie in its simplicity, the fact that it applies for general service requirements with finite second moments, and that it provides insights into the dependency of the performance on the system parameters. We show that the approximation for the mean (un)conditional sojourn time of a customer is decreasing as its relative priority increases. We also show that the approximation is exact in various scenarios, and that it is uniformly bounded in the second moments of the service requirements. Finally we numerically illustrate that the approximation is accurate across a broad range of parameters.
\end{abstract}

\section{INTRODUCTION}

The Discriminatory Processor Sharing queue (DPS) is a versatile queueing model providing a natural framework to model service differentiation in systems. It is a multi-class extension of the well-studied egalitarian Processor Sharing (PS) policy, where the various classes are assigned positive weight factors. The service capacity is shared simultaneously among all customers present in proportion to the respective class-dependent weights. More precisely, given there are $K$ classes of customers, if at time $t$ there are $n_{k}(t)$ class- $k$ customers present in the system, $k=1, \ldots, K$, under DPS each class- $k$ customer is served at rate $g_{k} / \sum_{j=1}^{K} g_{j} n_{j}(t)$, where $g_{1}, \ldots, g_{K}$, are the class-dependent weights. The DPS queue has received lot of attention due to its application to model the impact of service differentiation in systems.

When all the weights are equal, the DPS queue is equivalent to the PS queue. The PS queue has gained a prominent role in evaluating the performance of a variety of resource allocation mechanisms (see for example [18], [14], [27]), and in recent years it has received renewed attention as a convenient abstraction for modeling the flow-level performance of bandwidth-sharing protocols in packet-switched networks, in particular TCP, see for example [10], [23]. In multiple practical situations, the actual service shares that users obtain may show substantial variation among users with heterogeneous characteristics. For example, TCP flows that share a common bottleneck link but traverse distinct routes, may experience diverse packet loss rates and round-trip delays. Besides TCPrelated effects, the heterogeneity in bandwidth shares may also be due to deliberate service differentiation among competing flows (for example different quality-of-service in the Internet). For instance packet scheduling algorithms, such as Weighted Fair Queueing (WFQ) and Weighted Round-Robin (WRR), have been proposed as potential instruments to implement differentiated bandwidth sharing.

In this context, the Discriminatory Processor-Sharing (DPS) provides a natural approach for modeling the flow-level performance of TCP. The DPS model was introduced by Kleinrock in [17]. Despite the simplicity of the model description and the fact that the properties of the egalitarian PS queue are quite thoroughly understood, the analysis of DPS has proven to be extremely difficult. For example, results on an important basic metric like the mean sojourn time in the system have only been derived in a very implicit manner or under certain limiting regimes (time-scale decomposition, heavy-traffic, overload etc.).

In a seminal paper Fayolle, Mitrani \& Iasnogorodski [9] studied the mean conditional (on the service requirement) and unconditional sojourn time. For general service time distributions, the authors obtained the mean conditional sojourn time as the solution of a system of integro-differential equations. In addition, the authors provided a thorough analysis for the case of exponentially distributed service requirements. However, except for the case of two classes, no closed-form expression is available and numerical analysis is needed in order to calculate the mean sojourn times. Since we use the results of [9] in order to evaluate the accuracy of our approximation, we will give further details on them in Section 2. Avrachenkov et al. [3] established that the mean queue lengths of all classes are finite under the usual stability condition, regardless of the higherorder moments of the service requirements. Asymptotics of the sojourn time have also received considerable attention for example in [5] and [4]. An important result in this area establishes the asymptotic equivalence between the sojourn time distribution and the service time distribution. Time-scale separations have been studied in [24] and [6]. In particular, the authors of [6] approximate the distribution of the sojourn time for a DPS queue with admission control. However the expressions derived in [6] need to be solved numerically. The 
performance of DPS in overload and its application to model TCP flows is considered in [2]. The application of DPS to analyse the performance of TCP is also considered in [16]. For more applications of DPS in communication networks see [7], [8], [13]. DPS under a heavy-traffic regime (when the traffic load approaches the available capacity) was analysed in Grishechkin [11] assuming finite second moments of the service requirement distributions. Subsequently, assuming exponential service requirement distributions, a direct approach to establish a heavy-traffic limit for the joint queue length distribution was described by Rege \& Sengupta [19] and extended to phasetype distributions in [25]. We refer to the next section for more details on heavy-traffic results. Game-theoretic aspects of DPS have been studied in [26] and [12]. For an extensive overview of the literature on DPS we refer to the survey [1].

Motivated by the difficulty in analyzing the system in exact form, in this paper we derive a closed-form approximation for the mean (un)conditional sojourn time in the system. We first obtain a light-traffic approximation using the framework obtained in [11]. To the best of our knowledge, we are the first to obtain a light-traffic approximation of a time-sharing system, that is, when all users in the system simultaneously get served. We then use results from the heavy-traffic literature in order to obtain a polynomial approximation for any value of the load of the mean conditional sojourn time for service requirements with finite second moments. Unconditioning on the service time distribution, this allows us to readily obtain an approximation for the mean unconditional sojourn time. We will show that in some cases our approximation becomes exact, namely when there is only one class in the system or when all the weights are the same. The approximation provides insights into the performance of the system. We show that the approximation for the mean conditional sojourn time of a class- $k$ user is decreasing (resp. increasing) as the weight $g_{k}$ (resp. $g_{j}, j \neq k$ ) increases. Another important observation is that the approximation is uniformly bounded in the second moments of the service requirements. This was a major property of PS, which is in sheer contrast with FCFS queues, where the mean waiting time explodes as the second moment grows. Finally, we numerically investigate the accuracy of the approximation by comparing it with the exact results obtained in [9]. We consider different service time distributions, and our results show that our approximation works extremely well across various parameter values. An important benefit of the approximation is that it provides insights into the dependency of the performance on the system parameters (weights, service time distributions, etc), and we thus believe it will provide an interesting tool in order to implement service-differentiation in real systems.

The remainder of the paper is organized as follows. In Section II we provide a detailed model description and gather results from Fayolle et al. [9] and Grishechkin [11] that will be used in the paper. In Section III we develop a light-traffic analysis. The light-traffic interpolation approximation for the (un)conditional sojourn time is presented in Section IV. In Section V we numerically test the accuracy of the obtained approximations.

\section{MODEL DESCRIPTION AND PRELIMINARIES}

We consider a multi-class single-server queue with $K$ classes of customers. Class- $k$ customers, $k=1, \ldots, K$, arrive according to independent Poisson processes with rate $\lambda_{k} \geq 0$. We denote the overall arrival rate by $\lambda=\sum_{k=1}^{K} \lambda_{k}$. A class$k$ customer has a generally distributed service requirement denoted by $B_{k}$ and we assume $\mathbb{E}\left[B_{k}^{2}\right]<\infty, k=1, \ldots, K$. The traffic intensity for class- $k$ customers is denoted by $\rho_{k}:=\lambda_{k} \mathbb{E}\left[B_{k}\right]$ and the total traffic intensity is denoted by

$$
\rho:=\sum_{k=1}^{K} \rho_{k}=\sum_{k=1}^{K} \lambda_{k} \mathbb{E}\left[B_{k}\right]=\lambda \sum_{k=1}^{K} \alpha_{k} \mathbb{E}\left[B_{k}\right]=\lambda \mathbb{E}[B],
$$

where $\alpha_{k}=\lambda_{k} / \lambda$ denotes the probability that an arrival is of class $k$ and the random variable $B$ is the service requirement of an arbitrary arriving customer.

The $K$ customer classes share a common resource of capacity one. There are strictly positive weights $g_{1}, \ldots, g_{K}$ associated with each of the classes. Whenever there are $n_{k}$ class- $k$ customers, $k=1, \ldots, K$, in the system, each class- $k$ customer is served at rate

$$
\frac{g_{k}}{\sum_{j=1}^{K} n_{j} g_{j}} \text {. }
$$

We denote by $S_{k}(\lambda, b)$ the conditional sojourn time of a tagged class- $k$ customer with a given service requirement $b$, when the arrival rate is $\lambda$. We are interested in approximating $\bar{S}_{k}(\lambda, b):=\mathbb{E}\left[S_{k}(\lambda, b)\right]$, the mean conditional sojourn time of the tagged class- $k$ customer. We further denote by $\bar{S}_{k}(\lambda):=$ $\int_{0}^{\infty} \bar{S}_{k}(\lambda, b) \mathrm{d} F_{k}(b)$, the mean unconditional sojourn time of the tagged class- $k$ customer, where $\mathbb{P}\left(B_{k} \leq b\right)=F_{k}(b)$ is the distribution function of $B_{k}$.

The analysis of DPS is extremely difficult compared to that of egalitarian PS, which arises as a special case when all $g_{k}$ are equal. Fayolle et al. [9] obtained that the derivatives of the mean conditional sojourn times of the various classes satisfy the following system of integro-differential equations:

$$
\begin{aligned}
& \bar{S}_{k}^{(1)}(\lambda, b) \\
& =1+\sum_{j=1}^{K} \int_{0}^{\infty} \lambda_{j} \frac{g_{j}}{g_{k}} \bar{S}_{j}^{(1)}(\lambda, y)\left[1-F_{j}\left(y+\frac{g_{j}}{g_{k}} b\right)\right] \mathrm{d} y \\
& \quad+\int_{0}^{b} \bar{S}_{k}^{(1)}(\lambda, y) \sum_{j=1}^{K} \lambda_{j} \frac{g_{j}}{g_{k}}\left[1-F_{j}\left(\frac{g_{j}}{g_{k}}(b-y)\right)\right] \mathrm{d} y,
\end{aligned}
$$

for $k=1, \ldots, K$, where $\bar{S}_{j}^{(1)}(\lambda, b):=\frac{\partial \bar{S}_{j}(\lambda, b)}{\partial \lambda}$. The natural boundary conditions are $\bar{S}_{k}(\lambda, 0)=0, k=1, \ldots, K$.

The only known analytical solution for this system of equations has been obtained under the assumption of exponentially distributed service requirements. In this case we denote by $\mu_{j}:=1 / \mathbb{E}\left[B_{j}\right], \forall j$. In [9] it is proved that

$$
\bar{S}_{k}(\lambda, b)=\frac{b}{1-\rho}+\sum_{j=1}^{m} \frac{g_{k} c_{j} \beta_{j}+d_{j}}{\beta_{j}^{2}}\left(1-\mathrm{e}^{-\beta_{j} b / g_{k}}\right)
$$

where $-\beta_{j}, j=1,2, \ldots, m$, are the $m$ distinct negative roots of

$$
\sum_{j=1}^{K} \frac{\lambda_{j} g_{j}}{\mu_{j} g_{j}+s}=1
$$


and where $c_{j}$ and $d_{j}, j=1, \ldots, m$, are a function of the input parameters and $\beta_{j}, j=1, \ldots, m$.

Furthermore, for the mean unconditional sojourn time with exponentially distributed service requirements, it is shown in [9] that $\bar{S}_{k}(\lambda), k=1, \ldots, K$, is the unique solution of the following system of equations:

$$
\bar{S}_{k}(\lambda)\left(1-\sum_{j=1}^{K} \frac{\lambda_{j} g_{j}}{\mu_{j} g_{j}+\mu_{k} g_{k}}\right)-\sum_{j=1}^{K} \frac{\lambda_{j} g_{j} \bar{S}_{j}(\lambda)}{\mu_{j} g_{j}+\mu_{k} g_{k}}=\frac{1}{\mu_{k}}
$$

A closed-form solution for this system of equations (4) is available only for the case of $K=2$, and is given by

$$
\bar{S}_{1}(\lambda)=\frac{1}{\mu_{1}(1-\rho)}\left(1+\frac{\mu_{1} \rho_{2}\left(g_{2}-g_{1}\right)}{\mu_{1} g_{1}\left(1-\rho_{1}\right)+\mu_{2} g_{2}\left(1-\rho_{2}\right)}\right),
$$

and

$$
\bar{S}_{2}(\lambda)=\frac{1}{\mu_{2}(1-\rho)}\left(1+\frac{\mu_{2} \rho_{1}\left(g_{1}-g_{2}\right)}{\mu_{1} g_{1}\left(1-\rho_{1}\right)+\mu_{2} g_{2}\left(1-\rho_{2}\right)}\right) .
$$

The above shows how hard and challenging it is to study analytically the DPS model. For this reason, as mentioned in the introduction, research has focused on analysing the DPS queue in limiting regimes, like tail asymptotics, heavy-traffic limits, fluid limits etc. In this paper, we take a different approach, and we develop light-traffic interpolation based approximations for $\bar{S}_{k}(\lambda, b)$ and $\bar{S}_{k}(\lambda)$. In the numerical section we will use Equations (2), (4)-(6) in order to numerically verify the accuracy of our light-traffic interpolation approximations.

The approximation is obtained by interpolating the mean sojourn times obtained in both a light-traffic regime and a heavy-traffic regime.

The light-traffic regime consists in letting $\rho \downarrow 0$, or equivalently $\lambda \downarrow 0$. Hence, it concerns the performance when the system is almost empty. No results are available for the DPS queue. Therefore, in Section III we analyze the mean conditional sojourn time in the light-traffic regime.

The heavy-traffic regime consists in letting $\rho \uparrow 1$, or equivalently $\lambda \uparrow 1 / \mathbb{E}[B]$. Hence, it concerns the performance when it is close to congestion. Heavy-traffic results have been obtained in [11], [19], [25]. For our analysis, we use the results by Grishechkin [11, Theorem 4.1] who studied a general M/G/1/GPS system of which our model is a particular case. In particular, for the DPS queue as studied in this paper Grishechkin derives the distribution of the conditional sojourn times, scaled by $1-\lambda \mathbb{E}[B]=1-\rho$, as $\lambda \uparrow 1 / \mathbb{E}[B]$. In particular, the mean of this distribution is given by

$$
\mathbb{E}\left[\lim _{\lambda \uparrow 1 / \mathbb{E}[B]}(1-\lambda \mathbb{E}[B]) S_{k}(\lambda, b)\right]=\frac{b}{g_{k}} \frac{\mathbb{E}\left[B^{2}\right]}{\sum_{j=1}^{K} \alpha_{j} \mathbb{E}\left[B_{j}^{2}\right] / g_{j}} .
$$

For our interpolation result, we are interested in $\lim _{\lambda \uparrow 1 / \mathbb{E}[B]}(1-\lambda \mathbb{E}[B]) \bar{S}_{k}(\lambda, b)=\lim _{\lambda \uparrow 1 / \mathbb{E}[B]}(1-$ $\lambda \mathbb{E}[B]) \mathbb{E}\left[S_{k}(\lambda, b)\right]$. Although we cannot verify that the limit and expectation can be interchanged, we use the expression in (7) as an approximation for $\lim _{\lambda \uparrow 1 / \mathbb{E}[B]}(1-\lambda \mathbb{E}[B]) \bar{S}_{k}(\lambda, b)$. Numerical experiments as performed in [25] indicate that indeed the limits can be interchanged.

\section{LIGHT-TRAFFIC ANALYSIS}

In this section we analyse the mean conditional sojourn time of the tagged class- $k$ customer under the light-traffic regime. The light-traffic regime concerns the performance of the system for small values of the arrival rate $\lambda$, i.e., when the system is almost empty. We will approximate $\bar{S}_{k}(\lambda, b)$ by a Taylor series expansion of $\bar{S}_{k}(\lambda, b)$ at $\lambda=0$. Assuming that the first $n$ derivatives of $\bar{S}_{k}(\lambda, b)$ with respect to $\lambda$ at $\lambda=0$ exist we have the following approximation for the mean conditional sojourn time of a class- $k$ customer when $\lambda$ is close to zero:

$$
\bar{S}_{k}^{L T}(\lambda, b):=\bar{S}_{k}(0, b)+\lambda \bar{S}_{k}^{(1)}(0, b)+\cdots+\frac{\lambda^{n}}{n !} \bar{S}_{k}^{(n)}(0, b) .
$$

Here $\bar{S}_{k}^{(m)}(0, b), m=1, \ldots$, denotes the $m$-th derivative of $\bar{S}_{k}(\lambda, b)$ with respect to $\lambda$ at $\lambda=0$, i.e., $\left.\frac{\partial^{m} \bar{S}_{k}(\lambda, b)}{\partial \lambda^{m}}\right|_{\lambda=0}$. In this paper we set $n=1$ in (8) as this will already provide us with an accurate approximation of the performance. We note that in previous literature higher order approximations have been obtained for non-preemptive systems, see [20].

We have based our analysis on Reiman and Simon [22] where it is shown how to obtain the derivatives of arbitrary order $m \geq 0$ at $\lambda=0$ under a general admissibility condition. It can be seen that our model, being based on an $M / G / 1$, satisfies this condition. Hence, we consider the system in steady state. We assume the system starts at $t=-\infty$, and $S_{k}$ is the sojourn time of the tagged class- $k$ customer who arrives in the system at time $t=0$. Using [22] we have $\bar{S}_{k}(0, b)=\bar{\psi}(\emptyset)$ and

$$
\bar{S}_{k}^{(1)}(0, b)=\int_{\mathbb{R}}(\bar{\psi}(\{t\})-\bar{\psi}(\emptyset)) \mathrm{d} t
$$

where

$$
\begin{aligned}
\bar{\psi}(\emptyset) & :=\mathbb{E}\left[S_{k} \mid \text { no arrivals on } \mathbb{R}\right] \\
\bar{\psi}(\{t\}) & :=\mathbb{E}\left[S_{k} \mid \text { exactly one arrival on } \mathbb{R} \text { at } t\right] .
\end{aligned}
$$

Equation (10) represents the situation where nobody enters the system except the tagged customer. Therefore, $\bar{\psi}(\emptyset)$ is equal to the service requirement of the tagged customer, which we denote by $b$. Hence,

$$
\bar{S}_{k}(0, b)=b \text {. }
$$

Regarding (11), we denote by $S_{k, t, u_{t}, b_{u_{t}}}$ the sojourn time of the tagged class- $k$ customer when there is exactly one arrival at time $t$ on $\mathbb{R}, u_{t}$ describing the class of the customer arriving at time $t$ and $b_{u_{t}}$ denoting the service requirement of the customer

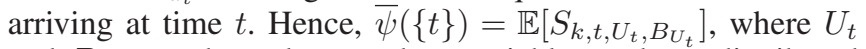
and $B_{U_{t}}$ are dependent random variables and are distributed as follows: with probability $\alpha_{i}$ we have $U_{t}=i$ and $B_{U_{t}}$ is distributed as $B_{i}, i=1, \ldots, K$. We can write $S_{k, t, u_{t}, b_{u_{t}}}$ as follows:

$$
\begin{aligned}
& S_{k, t, u_{t}, b_{u_{t}}=} \\
& \begin{cases}t+b_{u_{t}}+b & \text { if } t \leq 0 \leq t+b_{u_{t}} \text { and } \frac{b}{g_{k}}>\frac{t+b_{u_{t}}}{g_{u_{t}}} \\
\frac{g_{k}+g_{u_{t}}}{g_{k}} b & \text { if } t \leq 0 \leq t+b_{u_{t}} \text { and } \frac{b}{g_{k}} \leq \frac{t+b_{u_{t}}}{g_{u_{t}}} \\
b & \text { if } t+b_{u_{t}}<0 \\
b+b_{u_{t}} & \text { if } 0<t<b \text { and } \frac{b-t}{g_{k}}>\frac{b_{u_{t}}}{g_{u_{t}}} \\
t+(b-t) \frac{g_{k}+g_{u_{t}}}{g_{k}} & \text { if } 0<t<b \text { and } \frac{b-t}{g_{k}} \leq \frac{b_{u_{t}}}{g_{u_{t}}} \\
b & \text { if } 0<b<t,\end{cases}
\end{aligned}
$$


which can be seen as follows: The first expression describes the case where the customer arrives before the tagged customer and leaves after the tagged customer arrives, but before the tagged customer leaves. Hence, by the work conserving property, the tagged customer stays in the system until all the work present at time 0 is done, that is, $b_{u_{t}}-(-t)+b$. We recall that the work-conserving property states that as long as the system is non-empty, the server does not idle. The second term describes the case where the other customer is in the system at time 0 and is still present as the tagged customer departs. Hence, the tagged class- $k$ customer is served at rate $\frac{g_{k}}{g_{k}+g_{u_{t}}}$, so that its sojourn time is $b\left(\frac{g_{k}}{g_{k}+g_{u_{t}}}\right)^{-1}$. The fourth expression describes the case where the customer arrives after the tagged customer and leaves before the tagged customer. Hence, by the work-conserving property of the system, the sojourn time of the tagged class- $k$ customer is given by the total amount of work that needs to be done, that is, $b+b_{u_{t}}$. The fifth term describes the case where the customer arrives after the tagged customer, and departs after the tagged customer departs. Then, the sojourn time of the tagged customer is composed of $t$, the time it was in the system until the customer arrived, plus $(b-t)\left(\frac{g_{k}}{g_{k}+g_{u_{t}}}\right)^{-1}$, the remaining service requirement multiplied by the inverse of the rate at which the the tagged class- $k$ customer is served. The third and sixth case is when the tagged customer does not coincide with the other customer. Hence, the sojourn time is given by its service requirement, $b$.

From Equations (9) and (13) we then obtain the following expression for the first derivative.

Lemma III.1. We have

$$
\begin{aligned}
& \bar{S}_{k}^{(1)}(0, b) \\
& =\int_{\mathbb{R}}(\bar{\psi}(\{t\})-\bar{\psi}(\emptyset)) \mathrm{d} t=\int_{\mathbb{R}}\left(\mathbb{E}\left[S_{k, t, U_{t}, B_{U_{t}}}\right]-b\right) \mathrm{d} t \\
& =\mathbb{E}\left[\frac{1}{2}\left(1+\frac{g_{k}}{g_{U_{t}}}\right) \min \left\{B_{U_{t}}, b \frac{g_{U_{t}}}{g_{k}}\right\}^{2}\right. \\
& \left.-\left(b \frac{g_{U_{t}}}{g_{k}}+\frac{g_{k}}{g_{U_{t}}} B_{U_{t}}\right) \min \left\{B_{U_{t}}, b \frac{g_{U_{t}}}{g_{k}}\right\}+\frac{g_{k}+g_{U_{t}}}{g_{k}} b B_{U_{t}}\right] \text {. }
\end{aligned}
$$

Proof: To calculate $\bar{S}_{k}^{(1)}(0, b)$ we need to calculate

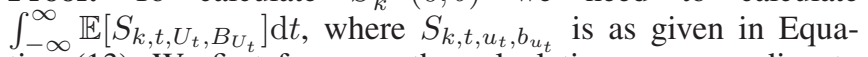
tion (13). We first focus on the calculation corresponding to the first term of (13), that is, the case when $t \leq 0 \leq t+B_{U_{t}}$ and $t<\frac{g_{U_{t}}}{g_{k}} b-B_{U_{t}}$, (where the inequalities of the random variables hold sample-path wise). We have

$$
\begin{aligned}
& \int_{-\infty}^{0} \mathbb{E}\left[\mathbf{1}\left[-B_{U_{t}} \leq t<\frac{g_{U_{t}}}{g_{k}} b-B_{U_{t}}\right]\left(t+B_{U_{t}}+b\right)\right] \mathrm{d} t \quad \begin{array}{l}
\text { In this section we present the light-traffic interpolation result. } \\
\text { This technique was popularized by Reiman and Simon [20], } \\
\text { [21], [22] and consists in interpolating }
\end{array} \\
& =\int_{0}^{\infty} \mathbb{E}\left[\mathbf{1}\left[B_{U_{t}} \geq t>B_{U_{t}}-\frac{g_{U_{t}}}{g_{k}} b\right]\left(-t+B_{U_{t}}+b\right)\right] \mathrm{d} t \quad t_{k}(\lambda):=(1-\rho) \bar{S}_{k}(\lambda, b)=(1-\lambda \mathbb{E}[B]) \bar{S}_{k}(\lambda, b) \\
& =\mathbb{E}\left[\int_{0}^{\infty} \mathbf{1}\left[B_{U_{t}} \geq t>B_{U_{t}}-\frac{g_{U_{t}}}{g_{k}} b\right]\left(-t+B_{U_{t}}+b\right) \mathrm{d} t\right], \quad \begin{array}{c}
\text { by a polynomial } \hat{t}_{k}(\lambda) \text { of order } n+1: \\
\hat{t}_{k}(\lambda)=h_{0}+h_{1} \lambda+\ldots+h_{n+1} \lambda^{n+1}
\end{array}
\end{aligned}
$$
we will show in Section IV that (16) is decreasing in $g_{k}$ and increasing in $g_{j}, j \neq k$. In other words, the approximation for the mean sojourn time reduces as its own weight increases, and it increases as the weight of any other class increases. Another interesting observation is that the light-traffic approximation of the mean conditional sojourn time can be uniformly bounded in the second moment. This important feature helps obtaining a good performance in the presence of highly variable service distributions (like the ones observed in nowadays communication systems). See Section IV-D for details.

\section{LIGHT-TRAFFIC INTERPOLATION}

We can infer several nice properties from (16). For instance, 
To determine the coefficients $h_{0}, \ldots, h_{n}$ we use the so-called light-traffic conditions: $\hat{t}_{k}(0)=t_{k}(0)$, and

$$
\hat{t}_{k}^{(m)}(0)=t_{k}^{(m)}(0), \text { for } m=1, \ldots, n,
$$

and the heavy-traffic condition

$$
\hat{t}_{k}\left((1 / \mathbb{E}[B])^{-}\right)=t_{k}\left((1 / \mathbb{E}[B])^{-}\right),
$$

where $t_{k}\left((1 / \mathbb{E}[B])^{-}\right)$is given by $\frac{b}{g_{k}} \frac{\mathbb{E}\left[B^{2}\right]}{\sum_{j=1}^{J} \alpha_{j} \mathbb{E}\left[B_{j}^{2}\right] / g_{j}}$, see (7). Once we have obtained the coefficients we undo the normalization so that

$$
\bar{S}_{k}^{I N T}(\lambda, b):=\frac{\hat{t}_{k}(\lambda)}{(1-\lambda \mathbb{E}[B])}, \quad 0 \leq \lambda<1 / \mathbb{E}[B]
$$

provides an approximation for the mean conditional sojourn time $\bar{S}_{k}(\lambda, b)$. We refer to this approximation as the lighttraffic interpolation of order $n+1$.

Note that in the previous section we derived the light-traffic derivative of order $1, \bar{S}_{k}^{(1)}(0, b)$. Hence, this allows us to obtain the light-traffic interpolation of order 2 as stated in the following proposition.

Proposition IV.1. The light-traffic interpolation (of order 2) of the mean conditional sojourn time for a tagged class- $k$ customer with service requirement $b$ is given by

$$
\begin{aligned}
& \bar{S}_{k}^{I N T}(\lambda, b) \\
&= b(1+\rho)+\lambda \mathbb{E}\left[\frac{1}{2}\left(1+\frac{g_{k}}{g_{U_{t}}}\right) \min \left\{B_{U_{t}}, b \frac{g_{U_{t}}}{g_{k}}\right\}^{2}\right. \\
&\left.-\left(b \frac{g_{U_{t}}}{g_{k}}+\frac{g_{k}}{g_{U_{t}}} B_{U_{t}}\right) \min \left\{B_{U_{t}}, b \frac{g_{U_{t}}}{g_{k}}\right\}+b \frac{g_{U_{t}}}{g_{k}} B_{U_{t}}\right] \\
&+\frac{(\lambda \mathbb{E}[B])^{2}}{(1-\lambda \mathbb{E}[B])} \frac{b}{g_{k}} \frac{\mathbb{E}\left[B^{2}\right]}{\sum_{j=1}^{J} \alpha_{j} \mathbb{E}\left[B_{j}^{2}\right] / g_{j}} .
\end{aligned}
$$

Proof: We have $\hat{t}_{k}(0)=h_{0}$ and by Equation (12) $t_{k}(0)=\bar{S}_{k}(0, b)=b$. Hence, by the light-traffic conditions this implies $h_{0}=b$. We further have $\hat{t}_{k}^{(1)}(0)=$ $h_{1}$ and $t_{k}^{(1)}(0)=-\mathbb{E}[B] \bar{S}_{k}(0, b)+\bar{S}_{k}^{(1)}(0, b)$, where we have by Lemma III.1 that $\bar{S}_{k}^{(1)}(0, b)=\mathbb{E}\left[\frac{1}{2}(1+\right.$ $\left.\frac{g_{k}}{g_{U_{t}}}\right) \min \left\{B_{U_{t}}, b \frac{g_{U_{t}}}{g_{k}}\right\}^{2}-\left(b \frac{g_{U_{t}}}{g_{k}}+\frac{g_{k}}{g_{U_{t}}} B_{U_{t}}\right) \min \left\{B_{U_{t}}, b \frac{g_{U_{t}}}{g_{k}}\right\}+$ $\left.\frac{g_{k}+g_{U_{t}}}{g_{k}} b B_{U_{t}}\right]$. This implies $h_{1}=-b \mathbb{E}[B]+\frac{1}{2} \mathbb{E}[(1+$ $\left.\frac{g_{k}}{g_{U_{t}}}\right) \min \left\{B_{U_{t}}, b \frac{g_{U_{t}}}{g_{k}}\right\}^{2}-\left(b \frac{g_{U_{t}}}{g_{k}}+\frac{g_{k}}{g_{U_{t}}} B_{U_{t}}\right) \min \left\{B_{U_{t}}, b \frac{g_{U_{t}}}{g_{k}}\right\}+$ $\left.b \frac{g_{k}+g_{U_{t}}}{g_{k}} B_{U_{t}}\right]$.

We have $\hat{t}_{k}(1 / \mathbb{E}[B])=h_{0}+h_{1} / \mathbb{E}[B]+h_{2} / \mathbb{E}[B]^{2}$ and by (7) we have $t_{k}(1 / \mathbb{E}[B]) \approx \frac{b}{g_{k}} \frac{\mathbb{E}\left[B^{2}\right]}{\sum_{j=1}^{k} \alpha_{j} \mathbb{E}\left[B_{j}^{2}\right] / g_{j}}$. Hence, from (19) we obtain $h_{2}=\frac{b}{g_{k}} \frac{\mathbb{E}[B]^{2} \mathbb{E}\left[B^{2}\right]}{\sum_{j=1}^{K} \alpha_{j} \mathbb{E}\left[B_{j}^{2}\right] / g_{j}}-$ $\mathbb{E}[B]\left(\frac{1}{2} \mathbb{E}\left[\left(1+\frac{g_{k}}{g_{U_{t}}}\right) \min \left\{B_{U_{t}}, b \frac{g_{U_{t}}}{g_{k}}\right\}^{2}\right]-\mathbb{E}\left[\left(b \frac{g_{U_{t}}}{g_{k}}+\right.\right.\right.$
$\left.\left.\left.\frac{g_{k}}{g_{U_{t}}} B_{U_{t}}\right) \min \left\{B_{U_{t}}, b \frac{g_{U_{t}}}{g_{k}}\right\}\right] \quad+\quad b \mathbb{E}\left[\frac{g_{k}+g_{U_{t}}}{g_{k}} B_{U_{t}}\right]\right)$. The final expression (21) is then obtained from $\left(h_{0}+h_{1} \lambda+h_{2} \lambda^{2}\right) /(1-\lambda \mathbb{E}[B])$ after a few manipulations.

From Proposition IV.1 we can make the following observations.

\section{A. The case of PS}

For the standard Processor Sharing queue the mean conditional sojourn time is known and is given by $b /(1-\rho)$, [15]. If either (i) there is only one class or (ii) all weights are the same, our model is equivalent to a processor-sharing queue. Below we will verify that our approximation as stated in (21) indeed coincides with $b /(1-\rho)$.

We first consider the case of one class, that is, $\alpha_{i}=0, \forall i \neq k$ and $\alpha_{k}=1$. Then Equation (21) is equal to

$$
\begin{aligned}
& b(1+\rho)+\lambda_{k} \mathbb{E}\left[\min \left\{B_{U_{t}}, b\right\}^{2}-\left(b+B_{U_{t}}\right) \min \left\{B_{U_{t}}, b\right\}\right. \\
& \left.+b B_{U_{t}}\right]+b \frac{\rho^{2}}{(1-\rho)}=b\left(1+\rho+\frac{\rho^{2}}{(1-\rho)}\right)=\frac{b}{1-\rho},
\end{aligned}
$$

where we used that $\min \left\{B_{U_{t}}, b\right\}^{2}-\left(b+B_{U_{t}}\right) \min \left\{B_{U_{t}}, b\right\}+$ $b B_{U_{t}}=0$.

We now assume all weights are the same, i.e., $g_{i}=g_{k}, \forall i, k=$ $1, \ldots, K$. Equation (21) is then equal to

$$
\begin{gathered}
b(1+\rho)+\lambda \mathbb{E}\left[\min \left\{B_{U_{t}}, b\right\}^{2}-\left(b+B_{U_{t}}\right) \min \left\{B_{U_{t}}, b\right\}+b B_{U_{t}}\right] \\
+\frac{b \rho^{2}}{(1-\rho)} \frac{\mathbb{E}\left[B^{2}\right]}{\sum_{j=1}^{K} \alpha_{j} \mathbb{E}\left[B_{j}^{2}\right]}=b\left(1+\rho+\frac{\rho^{2}}{(1-\rho)}\right)=\frac{b}{1-\rho} .
\end{gathered}
$$

Hence, both cases coincide with the PS queue.

\section{B. Priority queиe}

We now consider the case when the weight of the tagged customer grows large, i.e., $g_{k} \rightarrow \infty$. Hence, class $k$ is prioritized in the limit. Then, the approximation simplifies to

$$
\begin{aligned}
& \lim _{g_{k} \rightarrow \infty}[b(1+\rho) \\
& +\sum_{u_{t}=1}^{K} \lambda_{u_{t}} \mathbb{E}\left[\frac{1}{2}\left(\min \left\{B_{u_{t}}, b \frac{g_{u_{t}}}{g_{k}}\right\}^{2}+\frac{g_{k}}{g_{u_{t}}} \min \left\{B_{u_{t}}, b \frac{g_{u_{t}}}{g_{k}}\right\}^{2}\right)\right. \\
& \left.-\left(b \frac{g_{u_{t}}}{g_{k}} \min \left\{B_{u_{t}}, b \frac{g_{u_{t}}}{g_{k}}\right\}+B_{u_{t}} \min \left\{B_{u_{t}} \frac{g_{k}}{g_{u_{t}}}, b\right\}\right)+\frac{g_{u_{t}}}{g_{k}} b B_{u_{t}}\right] \\
& \left.+\frac{b(\lambda \mathbb{E}[B])^{2}}{(1-\lambda \mathbb{E}[B])} \frac{\mathbb{E}\left[B^{2}\right]}{g_{k} \sum_{j=1}^{K} \alpha_{j} \mathbb{E}\left[B_{j}^{2}\right] / g_{j}+\alpha_{k} \mathbb{E}\left[B_{k}^{2}\right]}\right] \\
& =b(1+\rho)+\mathbb{E}\left[\sum_{\substack{j \neq k \\
u_{t}=1}}^{K} \lambda_{u_{t}}\left(\frac{1}{2}(0+0)-\left(0+b B_{u_{t}}\right)+0\right)\right] \\
& =b\left(1+\rho_{k}\right) .
\end{aligned}
$$


Note that the conditional sojourn time as $g_{k} \rightarrow \infty$ is known and its given by $b /\left(1-\rho_{k}\right)$. Since $1 /\left(1-\rho_{k}\right)=\sum_{i=0}^{\infty} \rho_{k}^{i}$, we directly see that the approximation is the first order approximation of the exact expression. The relative error is equal to $100 \%\left(b /\left(1-\rho_{k}\right)-b\left(1+\rho_{k}\right)\right) / b /\left(1-\rho_{k}\right)=\rho_{k}^{2} 100 \%$, and we thus see that the relative error increases as the load of class $k$ increases.

\section{Monotonicity in the weights}

It can be checked that the approximation for the mean conditional sojourn time of a tagged class- $k$ customer, $\bar{S}_{k}^{I N T}(\lambda, b)$, is decreasing in $g_{k}$ and increasing in $g_{i}, i \neq k$.

This can be seen as follows. Conditioning on $U_{t}$ we can write

$$
\begin{aligned}
\bar{S}_{k}^{I N T}(\lambda, b) & b(1+\rho)+\sum_{i=1, i \neq k}^{K} \lambda_{i} \mathbb{E}\left[\frac{1}{2}\left(1+\frac{g_{k}}{g_{i}}\right) \min \left\{B_{i}, b \frac{g_{i}}{g_{k}}\right\}^{2}\right. \\
& \left.-\left(b \frac{g_{i}}{g_{k}}+\frac{g_{k}}{g_{i}} B_{i}\right) \min \left\{B_{i}, b \frac{g_{i}}{g_{k}}\right\}+b \frac{g_{i}}{g_{k}} B_{i}\right] \\
& +\frac{(\lambda \mathbb{E}[B])^{2}}{(1-\lambda \mathbb{E}[B])} \frac{b}{g_{k}} \frac{\mathbb{E}\left[B^{2}\right]}{\sum_{j=1}^{K} \alpha_{j} \mathbb{E}\left[B_{j}^{2}\right] / g_{j}},
\end{aligned}
$$

where for $U_{t}=k$ we used that $\min \left\{B_{k}, b\right\}^{2}-(b+$ $\left.B_{k}\right) \min \left\{B_{k}, b\right\}+b B_{k}=0$.

Now, if $B_{i} \leq \frac{g_{i}}{g_{k}} b$, then $\frac{1}{2}\left(1+\frac{g_{k}}{g_{i}}\right) \min \left\{B_{i}, b \frac{g_{i}}{g_{k}}\right\}^{2}-\left(b \frac{g_{i}}{g_{k}}+\right.$ $\left.\frac{g_{k}}{g_{i}} B_{i}\right) \min \left\{B_{i}, b \frac{g_{i}}{g_{k}}\right\}+b \frac{g_{i}}{g_{k}} B_{i} \stackrel{g_{i}}{=} \frac{1}{2} B_{i}^{2}\left(1-\frac{g_{k}}{g_{i}}\right)$, which is decreasing in $g_{k}$ and increasing in $g_{i}$. If $B_{i}>\frac{g_{i}}{g_{k}} b$, then $\frac{1}{2}\left(1+\frac{g_{k}}{g_{i}}\right) \min \left\{B_{i}, b \frac{g_{i}}{g_{k}}\right\}^{2}-\left(b \frac{g_{i}}{g_{k}}+\frac{g_{k}}{g_{i}} B_{i}\right) \min \left\{B_{i}, b \frac{g_{i}}{g_{k}}\right\}+$ $b \frac{g_{i}}{g_{k}} B_{i}=\frac{1}{2} b^{2} \frac{g_{i}}{g_{k}}\left(1-\frac{g_{i}}{g_{k}}\right)+b B_{i}\left(\frac{g_{i}}{g_{k}}-1\right)$, which is decreasing in $g_{k}$ and increasing in $g_{i}$ (can be derived by taking the derivative and the fact that $\left.B_{i}>\frac{g_{i}}{g_{k}} b\right)$. The monotonicity of $\bar{S}_{k}^{I N T}(\lambda, b)$ in $g_{k}$ and $g_{i}$ now follows immediately.

\section{Uniformly bounded in the second moment}

A very relevant property of processor sharing is that the mean sojourn time depends on the service time distribution only through its mean [14]. This has been an important argument to claim the interest of time-sharing disciplines with respect to more classical scheduling policies like FCFS. Indeed, the classical Pollaczek-Khinchine formula for the mean waiting time in a FCFS queue shows that it explodes as the second moment of the service time distribution grows large. For a DPS queue, Equation (1) does not allow to reach any conclusion regarding the dependence of the mean conditional sojourn time on the moments of the service time distribution.

It then becomes interesting to observe that the approximation (21) is uniformly bounded in the second moments of the service time distribution. To see this, we first note that $\min \left\{B_{U_{t}}, b \frac{g_{U_{t}}}{g_{k}}\right\}^{2} \leq B_{U_{t}} b \frac{g_{U_{t}}}{g_{k}}$, which directly implies that the first three terms in (21) are uniformly bounded by a function that depends on the service requirements only through its first moment. We are now left with the heavy-traffic term $\frac{\mathbb{E}\left[B^{2}\right]}{\sum_{j=1}^{K} \alpha_{j} \mathbb{E}\left[B_{j}^{2}\right] / g_{j}}$. Let $j^{*}$ be such that $\mathbb{E}\left[B_{j^{*}}^{2}\right] \geq \mathbb{E}\left[B_{j}^{2}\right]$, $\forall j$. We then have $\frac{\mathbb{E}\left[B^{2}\right]}{\sum_{j=1}^{K} \alpha_{j} \mathbb{E}\left[B_{j}^{2}\right] / g_{j}}=\frac{\sum_{j} \alpha_{j} \mathbb{E}\left[B_{j}^{2}\right]}{\sum_{j=1}^{K} \alpha_{j} \mathbb{E}\left[B_{j}^{2}\right] / g_{j}} \leq$ $\frac{\mathbb{E}\left[B_{j^{*}}^{2}\right]}{\alpha_{j^{*}} \mathbb{E}\left[B_{j^{*}}^{2}\right] / g_{j^{*}}}=\frac{g_{j^{*}}}{\alpha_{j^{*}}}$. We thus finally conclude that (21) can be upper bounded by an expression that depends only on the first moment of the service time distributions. This indicates that the DPS queue provides a satisfactory performance even in the presence of service time distributions with a high variability.

\section{E. Mean unconditional sojourn time}

As a corollary of Proposition IV.1, we obtain the mean unconditional sojourn time of the tagged class- $k$ customer.

Corollary IV.2. The light-traffic interpolation (of order 2) of the mean unconditional sojourn time for a tagged class$k$ customer is given by

$$
\begin{aligned}
& \bar{S}_{k}^{I N T}(\lambda):=\int_{0}^{\infty} \bar{S}_{k}^{I N T}(\lambda, b) \mathrm{d} F_{k}(b) \\
& =\mathbb{E}\left[B_{k}\right](1+\rho) \\
& \quad+\lambda \mathbb{E}\left[\frac{1}{2}\left(1+\frac{g_{k}}{g_{U_{t}}}\right) \min \left\{B_{U_{t}}, B_{k} \frac{g_{U_{t}}}{g_{k}}\right\}^{2}\right. \\
& \left.\quad-\left(B_{k} \frac{g_{U_{t}}}{g_{k}}+\frac{g_{k}}{g_{U_{t}}} B_{U_{t}}\right) \min \left\{B_{U_{t}}, B_{k} \frac{g_{U_{t}}}{g_{k}}\right\}+B_{k} \frac{g_{U_{t}}}{g_{k}} B_{U_{t}}\right] \\
& \quad+\frac{(\lambda \mathbb{E}[B])^{2}}{(1-\lambda \mathbb{E}[B])} \frac{\mathbb{E}\left[B_{k}\right]}{g_{k}} \frac{\mathbb{E}\left[B^{2}\right]}{\sum_{j=1}^{K} \alpha_{j} \mathbb{E}\left[B_{j}^{2}\right] / g_{j}} .
\end{aligned}
$$

\section{NUMERICAL RESULTS}

In this section we numerically investigate the accuracy of the approximations obtained in this paper. In Section V-A we consider the mean conditional sojourn time and in Section V-B the mean unconditional sojourn time, whose approximations are stated in Proposition IV.1 and Corollary IV.2, respectively.

As stated in Section II, Fayolle et al. [9] obtain analytical expressions of the mean conditional and unconditional sojourn time under the assumption of exponentially distributed service requirements. We recall that a random variable $B_{i}$ is exponentially distributed if $\mathbb{P}\left(B_{i} \leq b_{i}\right)=1-\mathrm{e}^{\left(-b_{i} / \mathbb{E}\left[B_{i}\right]\right)}$. For exponentially distributed service requirements, we will evaluate the accuracy of the approximations by comparing the exact formulas as obtained in [9], see Equations (2) and (4), with the approximations as given in (21) and (22).

In order to obtain a more complete understanding on the accuracy of the approximation, we will also consider hyperexponential distributions. We say that $B_{i}$ has a hyperexponential distribution with $m_{i}$ phases if $\mathbb{P}\left(B_{i} \leq b_{i}\right)=$ $1-\sum_{k=1}^{m_{i}} p_{i k} \mathrm{e}^{\left(-b_{i} / \mathbb{E}\left[B_{i k}\right]\right)}$, where $p_{i k}$ is the probability that a class- $i$ customer is exponentially distributed with mean $\mathbb{E}\left[B_{i k}\right]$. Hyperexponential distributions have a decreasing hazard-rate, and their second moment can be made arbitrarily large, and because of these features it has been proposed as an appropriate distribution to model service time distributions in the Internet. In order to derive exact expressions for the mean sojourn time when the service requirements are hyperexponentially distributed, we make the observation that if classes $k=1, \ldots, m_{i}$ are exponentially distributed (where class $k$ has arrival rate $\lambda_{k}$ and mean service requirement $\mathbb{E}\left[B_{k}\right]$ ) and have the same 
DPS weight, $g_{1}=\ldots=g_{m_{i}}$, then they can be seen as a single (merged) class $i$ with a hyperexponential distribution with parameters $p_{i k}=\lambda_{k} / \sum_{l=1}^{m_{i}} \lambda_{l}$ and $\mathbb{E}\left[B_{i k}\right]=\mathbb{E}\left[B_{k}\right]$, for each phase $k=1, \ldots, m_{i}$.

Throughout this section the performance criteria will be the relative error. For instance, for the mean conditional sojourn time, we will calculate $100 \% \times \frac{\bar{S}_{k}(\lambda, b)-\bar{S}_{k}^{I N T}(\lambda, b)}{\bar{S}_{k}(\lambda, b)}$, and for the mean unconditional sojourn time $100 \% \times \frac{\bar{S}_{k}(\lambda)-\bar{S}_{k}^{I N T}(\lambda)}{\bar{S}_{k}(\lambda)}$.

Before explaining in detail the numerical results we have obtained, we summarize our main conclusions:

- The approximation is accurate over a broad range of parameter values.

- For a given set of parameters, the relative error for the mean conditional sojourn time increases as the service requirement of the tagged customer increases.

- The error increases as the disparity among the weights increases.

- For any given scenario, the largest relative error occurs in an intermediate load between 0 and 1 .

- The largest relative errors for the mean conditional sojourn time occur for service requirements $b$ that are very unlikely to occur. This also explains the high accuracy of our approximation for the mean unconditional sojourn time.

\section{A. Conditional sojourn time}

In this section we measure the accuracy of the mean conditional sojourn time.

Scenario 1. In Figure I we consider four classes $K=4$ with exponentially distributed service requirements. The parameters of the classes are fixed, and we vary the total arrival rate in order for the load to cover the range of stable values. We consider $\mathbb{E}\left[B_{1}\right]=2, \mathbb{E}\left[B_{2}\right]=5, \mathbb{E}\left[B_{3}\right]=7, \mathbb{E}\left[B_{4}\right]=10$, $g_{1}=30, g_{2}=25, g_{3}=20, g_{4}=10$, and $\alpha_{1}=10 / 36$, $\alpha_{2}=5 / 36, \alpha_{3}=8 / 36, \alpha_{4}=13 / 36$ such that $\lambda_{i}=\alpha_{i} * \lambda, i=$ $1, \ldots, 4$, where $\lambda$ is the total arrival rate. In Figure I we plot the relative error of our approximation for the mean conditional sojourn time of a tagged class- $i$ customer, for $i=1, \ldots, 4$, where the size of the tagged class- $i$ customer, $b_{i}$, is selected such that the probability of the event is $\mathbb{P}\left(B_{i} \leq b_{i}\right)=0.01$, $\mathbb{P}\left(B_{i} \leq b_{i}\right)=0.50$ and $\mathbb{P}\left(B_{i} \leq b_{i}\right)=0.99$, respectively. As can be seen, the relative error for the mean conditional sojourn time remains small and always below $6 \%$.

Scenario 2. In Figure II we consider two classes $K=2$ with exponentially distributed service requirements. We fixed the parameters $\mathbb{E}\left[B_{1}\right]=2, \mathbb{E}\left[B_{2}\right]=1, g_{1}=1, g_{2}=3$, $\alpha_{1}=0.415, \alpha_{2}=0.585$ and $\lambda_{i}=\alpha_{i} * \lambda$. We let the service requirement of the class- $i$ tagged customer span between 0 and $b_{i, \max }$ where $\mathbb{P}\left(B_{i} \leq b_{\mathrm{i}, \max }\right)=0.99$ and for each $b$ we plot the largest absolute relative error that can be found for a $\rho \in[0,1)$. We observe a largest error of at most $6 \%$.

Scenario 3. In Figure III we consider again two classes with exponentially distributed service requirements. As parameters we fix: $\mathbb{E}\left[B_{1}\right]=2, \mathbb{E}\left[B_{2}\right]=1, \lambda_{1}=0.2, \lambda_{2}=1.5 \lambda_{1}$ and $b=$

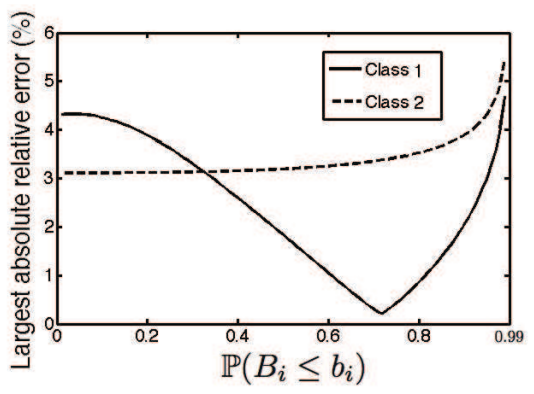

Figure II: Scenario 2. Largest absolute relative error as a function of $\mathbb{P}\left(B_{i} \leq b_{i}\right)$.

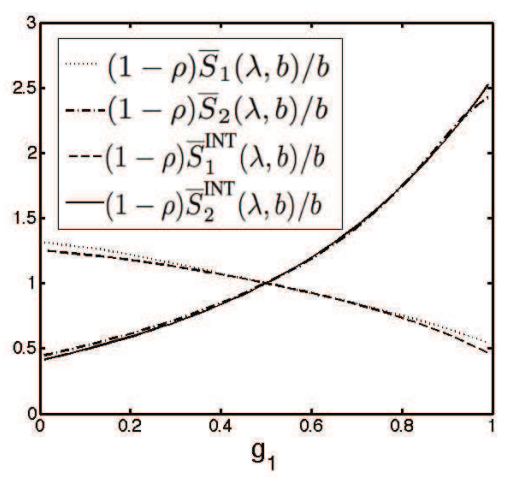

Figure III: Scenario 3: Mean conditional sojourn time as a function of $g_{1}$

1. We chose $g_{2}=1-g_{1}$ and let $g_{1}$ vary on the horizontal axis. In the figure we plot the mean conditional sojourn time and our approximation. We see that the property stated in Section IV-C is satisfied, namely as $g_{1}$ increases $\bar{S}_{1}^{I N T}(\lambda, b)$ decreases and $\bar{S}_{2}^{I N T}(\lambda, b)$ increases. Besides, it can be observed from the figure that the approximation looses accuracy as one class is given more priority, i.e., $g_{1} \rightarrow 0$ or $g_{1} \rightarrow 1$.

Scenario 4. In Figure IV we consider two classes with hyperexponential distributed service requirements with $\mathbb{E}\left[B_{1}\right]=2$, $\mathbb{E}\left[B_{2}\right]=6$. Each of the hyperexponential distributions has 3 phases. The parameters are as follows: for class 1 we take $\mathbb{E}\left[B_{11}\right]=3.5, \mathbb{E}\left[B_{12}\right]=2, \mathbb{E}\left[B_{13}\right]=5, p_{11}=10 / 21$, $p_{12}=5 / 21, p_{13}=6 / 21$, and for class 2 we take $\mathbb{E}\left[B_{21}\right]=10$, $\mathbb{E}\left[B_{22}\right]=15, \mathbb{E}\left[B_{23}\right]=20, p_{21}=4 / 15, p_{22}=8 / 15$, $p_{23}=3 / 15$. The weights are set to $g_{1}=2$ and $g_{2}=5$. We assume that an arriving customer is of class 1 (class 2) with probability $\alpha_{1}=21 / 36\left(\alpha_{2}=15 / 36\right)$. As in Scenario 1 , we select the service requirement of the tagged customer such that $\mathbb{P}\left(B_{i} \leq b_{i}\right)=0.01,0.5$ and 0.99 . We see that the error increases as the size of the tagged customer increases. However it is remarkable how accurate the approximation is.

In Figure $\mathrm{V}$ we consider Scenario 4. We vary the service requirement of the class- $i$ tagged customer between 0 and $b_{i, \max }$ where $\mathbb{P}\left(B_{i} \leq b_{\mathrm{i}, \max }\right)=0.99$ and for each $b$ we plot the largest absolute relative error that can be found for a $\rho \in[0,1)$. We observe that the error increases as the size of the tagged customer increases. The largest absolute relative error is of the 

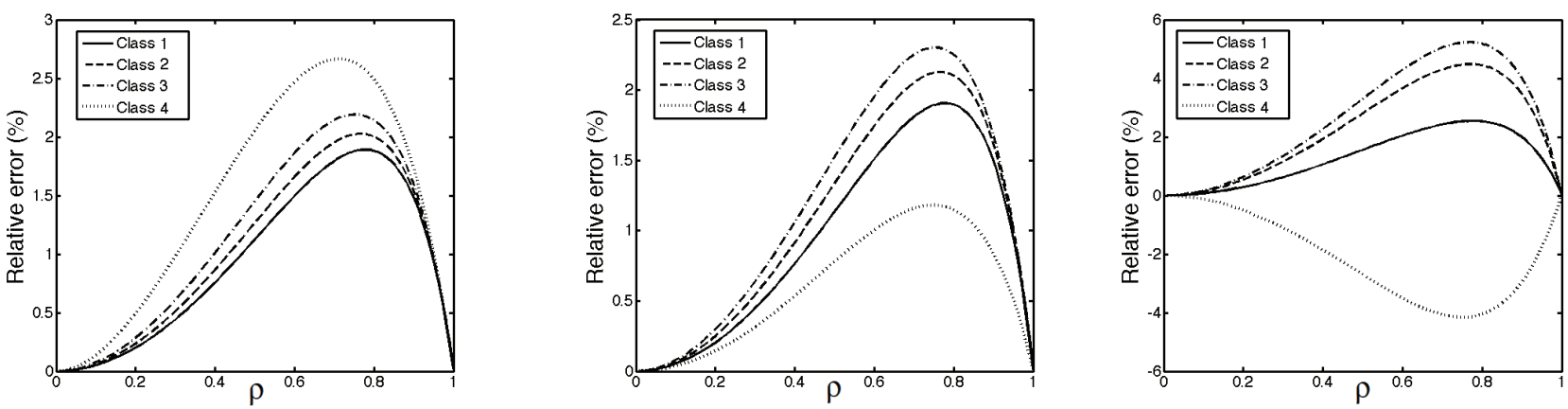

Figure I: Scenario 1: Relative error for a tagged class- $i$ customer with service requirement $b_{i}$ such that $\mathbb{P}\left(B_{i} \leq b_{i}\right)=0.01$ (left), $\mathbb{P}\left(B_{i} \leq b_{i}\right)=0.50$ (middle), $\mathbb{P}\left(B_{i} \leq b_{i}\right)=0.99$ (right).
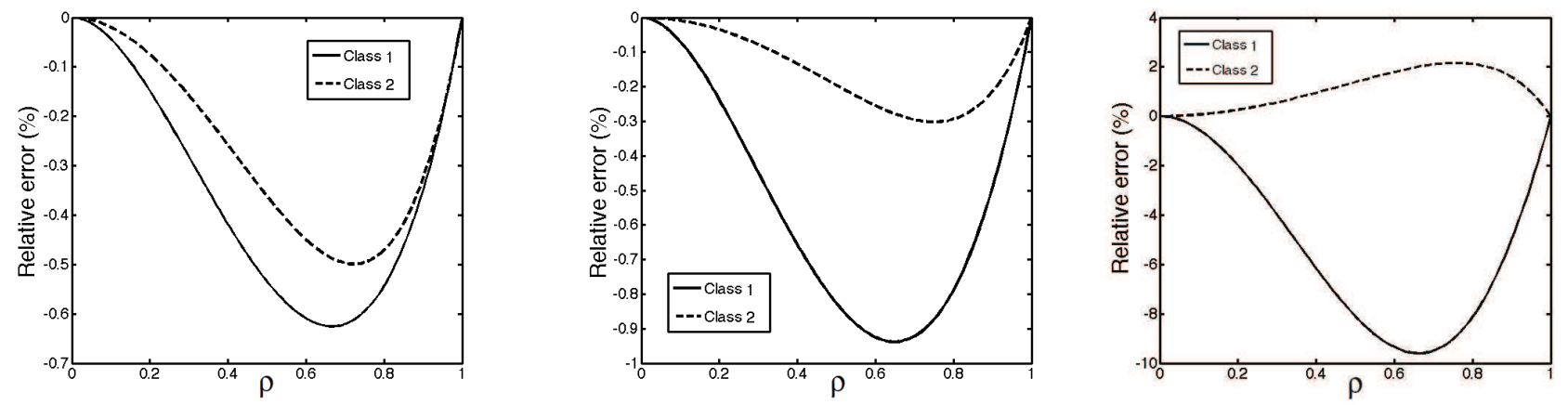

Figure IV: Scenario 4: Relative error for a tagged class- $i$ customer with service requirement $b_{i}$ such that $\mathbb{P}\left(B_{i} \leq b_{i}\right)=0.01$ (left), $\mathbb{P}\left(B_{i} \leq b_{i}\right)=0.50$ (middle), $\mathbb{P}\left(B_{i} \leq b_{i}\right)=0.99$ (right).

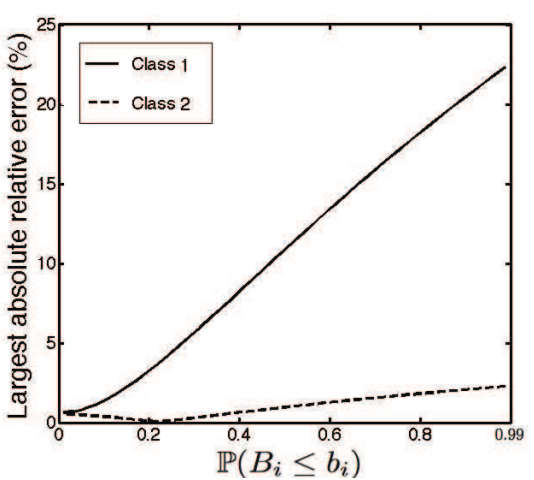

Figure V: Scenario 4. Largest absolute relative error as a function of $\mathbb{P}\left(B_{i} \leq b_{i}\right)$.

order of $22 \%$ for the class with the smallest weight and of the order of $3 \%$ for the class with the highest weight.

\section{B. Unconditional sojourn time}

In this section we evalute the accuracy of the mean unconditional sojourn time.

In Figure VI we consider the same parameter setting as in Scenario 1, and we observe that the largest relative error for the mean unconditional sojourn time is less than $3.5 \%$.

In Figure VII we consider two classes with hyper-exponentially distributed service requirements. The parameters are the ones considered in Scenario 4. We conclude that the largest relative error for the mean unconditional sojourn time is around $3 \%$.

As pointed out in the beginning of the section, we observe that the relative error for the mean unconditional sojourn time tends to be smaller than the ones observed for the mean conditional sojourn time. This can be explained by noting that the largest errors in the mean conditional sojourn time tend to occur for service requirements that happen with a very low probability.

In Figure VIII we consider two classes with hyperexponentially distributed service requirements. The parameters are the same as in Scenario 4 . We chose $g_{2}=1-g_{1}$ and let $g_{1}$ vary on the horizontal axis. For each given $g_{1}$ we calculate the largest absolute relative error for the mean unconditional sojourn time as we let $\rho$ range from 0 to 1 . We observe that the relative error for the unconditional sojourn time is at most of $30 \%$, and that this happens when class 2 receives full priority.

\section{ACKNOWLEDGEMENTS}

The authors are thankful to A. Makowski (University of Maryland at College Park) for helpful discussions. 


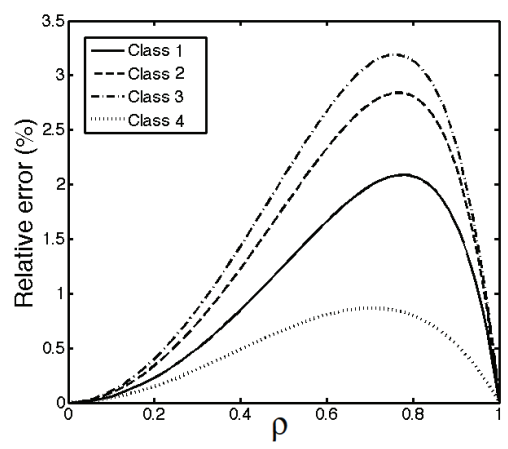

Figure VI: Relative error for the mean unconditional sojourn time in Scenario 1.

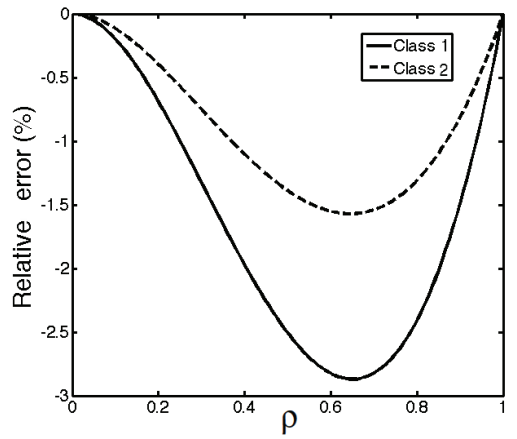

Figure VII: Relative error for the mean unconditional sojourn time in Scenario 4

\section{REFERENCES}

[1] E. Altman, K. Avrachenkov, and U. Ayesta. A survey on discriminatory processor sharing. Queueing systems, 53(1-2):53-63, 2006.

[2] E. Altman, T. Jimenez, and D. Kofman. DPS queues with stationary ergodic service times and the performance of TCP in overload. In Proceedings of IEEE INFOCOM, 2004.

[3] K.E. Avrachenkov, U. Ayesta, P. Brown, and R. Núñez-Queija. Discriminatory processor sharing revisited. In Proceedings of IEEE INFOCOM, 2005.

[4] S.C. Borst, R. Núñez-Queija, and A.P. Zwart. Sojourn time asymptotics

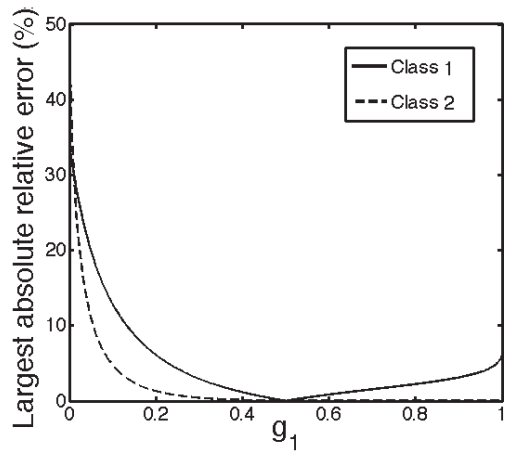

Figure VIII: Largest absolute relative error with respect to the weight $g_{1}$ in Scenario 4 in processor sharing queues. Queueing Systems, 53(1-2):31-51, 2006.

5] S.C. Borst, D.T.M.B. van Ooteghem, and A.P. Zwart. Tail asymptotics for discriminatory processor sharing queues with heavy-tailed service requirements. Performance Evaluation, 61(2-3):281-298, 2005.

[6] O.J. Boxma, N. Hegde, and R. Núñez-Queija. Exact and approximate analysis of sojourn times in finite discriminatory processor sharing queues. AEU International Journal on Electronic Communications, 60:109-115, 2006.

[7] T. Bu and D. Towsley. Fixed point approximation for TCP behaviour in an AQM network. In Proceedings of ACM SIGMETRICS/Performance, pages 216-225, 2001.

[8] S.K. Cheung, J.L. van den Berg, R.J. Boucherie, R. Litjens, and F. Roijers. An analytical packet/flow-level modelling approach for wireless LANs with quality-of-service support. In Proceedings of ITC19, 2005.

[9] G. Fayolle, I. Mitrani, and R. Iasnogorodski. Sharing a processor among many job classes. Journal of the ACM, 27(3):519-532, 1980.

[10] S. Ben Fredj, T. Bonald, A. Proutiere, G. Regnie, and J. Roberts. Statistical bandwidth sharing: A study of congestion at flow level. In SIGCOMM, pages 111-122, 2001.

[11] S. Grishechkin. On a relationship between processor sharing queues and Crump-Mode-Jagers branching processes. Adv. Appl. Prob., 24(3):653698, 1992.

[12] R. Hassin and M. Haviv. To Queue or not to Queue: Equilibrium Behavior in Queueing Systems. Kluwer Academic Publishers, Boston etc., 2003.

[13] Y. Hayel and B. Tuffin. Pricing for heterogeneous services at a discriminatory processor sharing queue. In Proceedings of Networking, 2005.

[14] F.P. Kelly. Stochastic Networks and Reversibility. Wiley, Chichester, 1979.

[15] F.P. Kelly. Charging and rate control for elastic traffic. European Transactions on Telecommunications, 8:33-37, 1997.

[16] A.A. Kherani and R. Núñez-Queija. TCP as an implementation of agebased scheduling: fairness and performance. In Proceedings of IEEE INFOCOM, 2006

[17] L. Kleinrock. Time-shared systems: A theoretical treatment. Journal of the ACM, 14(2):242-261, 1967.

[18] L. Kleinrock. Queueing Systems, vol. 2. John Wiley and Sons, 1976.

[19] K.M. Rege and B. Sengupta. Queue length distribution for the discriminatory processor sharing queue. Operations Research, 44(4):653-657, 1996.

[20] M.I. Reiman and B. Simon. An interpolation approximation for queueing systems with Poisson input. Operations Research, 36:454469, 1988.

[21] M.I. Reiman and B. Simon. Light traffic limits of sojourn time distributions in Markovian queueing networks. Stochastic Models, 4:191-233, 1988.

[22] M.I. Reiman and B. Simon. Open queueing systems in light traffic. Oper. Res., 14:26-59, 1989.

[23] J.W. Roberts. A survey on statistical bandwidth sharing. Computer Networks, 45:319-332, 2004

[24] G. van Kessel, R. Núñez-Queija, and S.C. Borst. Differentiated bandwidth sharing with disparate flow sizes. In Proceedings of IEEE INFOCOM, 2005.

[25] I.M. Verloop, U. Ayesta, and R. Núñez-Queija. Heavy-traffic analysis of a multiple-phase network with discriminatory processor sharing. Operations Research, 59(3):648-660, 2011.

[26] Yu Wu, Loc Bui, and Ramesh Johari. Heavy traffic approximation of equilibria in resource sharing games. IEEE Journal on Selected Areas in Communications, 30(11):2200-2209, 2012.

[27] S.F. Yashkov. Processor sharing queues: Some progress in analysis. Queueing Systems, 2:1-17, 1987. 